\title{
SORÇÃo E DESSORÇÃo do DiURON EM QUATro Latossolos BRASILEIROS $^{1}$
}

\author{
Sorption and Desorption of Diuron in Four Brazilian Latosols
}

ROCHA, P.R.R. ${ }^{2}$, FARIA, A.T. ${ }^{3}$, BORGES, L.G.F.C. ${ }^{4}$, SILVA, L.O.C. ${ }^{5}$, SILVA, A.A. ${ }^{6}$ e FERREIRA, E.A. ${ }^{7}$

\begin{abstract}
RESUMO - O conhecimento do processo de sorção de herbicidas nos solos é uma das condições para fazer recomendações seguras do ponto de vista técnico e ambiental desses compostos. Neste trabalho, foram avaliadas a sorção e dessorção do diuron em Latossolo VermelhoAmarelo (LVA), Latossolo Vermelho-Amarelo húmico (LVAh), Latossolo Vermelho (LV) e Latossolo Amarelo (LA), com diferentes valores de $\mathrm{pH}$. Na avaliação da sorção e dessorção utilizou-se o método Batch Equilibrium, que consistiu na utilização de $10,0 \mathrm{~mL}$ de solução com concentrações de 0,5, 1, 2, 4, 8 e $15 \mathrm{mg} \mathrm{L}^{-1}$ do diuron, preparadas em $\mathrm{CaCl}_{2}$ 0,01 $\mathrm{mol} \mathrm{L}^{-1}$. Essas soluções foram adicionadas a amostras de $2,00 \mathrm{~g}$ de solo, permanecendo sob agitação por 12 horas. Após centrifugação e filtração, a concentração do diuron no sobrenadante foi determinada por cromatografia liquida de alta eficiência. A dessorção foi avaliada utilizando as amostras contidas nos tubos, após os ensaios de sorção, que continham dose inicial de $8 \mathrm{mg} \mathrm{L}^{-1}$ de herbicida. A isoterma de Freundlich ajustou-se adequadamente para descrever a sorção do diuron em todos os solos estudados. A sorção do diuron foi diretamente relacionada aos teores de matéria orgânica, visto que solos com maiores teores de matéria orgânica, LVA e LVAh, apresentaram maiores coeficientes de sorção. O aumento do pH dos solos elevou a sorção do diuron, de forma mais evidente no LV, sendo este o solo que apresentou o menor teor de matéria orgânica. O processo de dessorção do diuron foi inverso ao da sorção, pois solos com menor sorção apresentaram as maiores porcentagens de dessorção.
\end{abstract}

Palavras-chave: herbicidas não iônicos, isotermas de Freundlich, pH do solo.

\begin{abstract}
The knowledge of herbicide sorption in soils is one of the conditions needed to make reliable recommendations of these compounds, based on theirtechnical and environmental aspects. Thus, the aim of this work was to evaluate sorption and desorption of diuron in Red-Yellow Latosol (RYL), Humic Red-Yellow Latosol (HRYL), Red Latosol (RL), and Yellow Latosol (YL), with different pH values. The Batch Equilibrium method was used to evaluate sorption and desorption. It consisted of the use of $10.0 \mathrm{~mL}$ of solution at concentrations of $0.5,1,2,4,8$, and $15 \mathrm{mg} \mathrm{L}^{-1}$ of diuron,

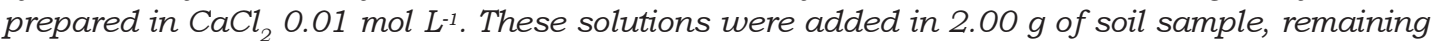
under agitation for $12 \mathrm{~h}$. After centrifugation and filtration, the diuron concentration in the supernatant was determined by high performance liquid chromatography. Desorption was evaluated using samples kept in tubes after the sorption tests, at an initial concentration of $8 \mathrm{mg} \mathrm{L}^{-1}$ of the herbicide. The Freundlich isotherm was adjusted appropriately to describe the sorption of diuron for all soils. The sorption of diuron was directly related to organic matter content, whereas soils with higher organic matter content, RYL and HRYL showed higher sorption coefficients. The $p H$ increase in soil increases diuron sorption. This was more evident in $R L$, which has the lowest organic matter content. The desorption process was the inverse of the diuron sorption process, since soils with lower sorption had the highest percentages of desorption.
\end{abstract}

Keywords: non-ionic herbicides, Freundlich isotherms, soil pH.

1 Recebido para publicação em 2.5.2012 e aprovado em 25.10.2012.

2 Engo-Agro ${ }^{-}$, D.Sc., Bolsista PNPD, Universidade Federal Rural do Semi-Árido - UFERSA, Mossoró-RN < paulo.rocha@ufv.br>; ${ }^{3}$ Eng-o-Agr $^{0}$., Mestrando no Programa de Pós-Graduação em Fitotecnia, Dep. de Fitotecnia, Universidade Federal de Viçosa - DFT/ UFV; ${ }^{4}$ Estudante de graduação do Curso de Agronomia, UFV, ${ }^{5}$ Química, D.Sc., Professora, Instituto Federal do Sudeste de Minas Gerais - Campus Barbacena, Barbacena-MG; ${ }^{6}$ Eng $^{\circ}-$ Agr $^{\circ}$., D.Sc. Professor, Associado, DFT/UFV; ${ }^{7}$ Eng $^{\circ}-$ Agr $^{\circ}$., D.Sc., Bolsista PNPD, Universidade Federal dos Vales do Jequitinhonha e Mucuri - UFVJM, Diamantina-MG.

Planta Daninha, Viçosa-MG, v. 31, n. 1, p. 231-238, 2013 


\section{INTRODUÇÃO}

A utilização dos agrotóxicos na agricultura é de fundamental importância para evitar perdas de produtividade das culturas, porém o uso incorreto desses compostos sintéticos representa riscos de contaminação ao meio ambiente. Entre os agrotóxicos, os herbicidas são os mais utilizados (SINDAG, 2011) e também os mais frequentemente encontrados em águas superficiais e subsuperficiais (Bacigalupo \& Meroni, 2007; Lapworth \& Gooddy, 2006).

A interação solo-herbicida altera a disponibilidade do produto na solução do solo e é governada pelos processos de retenção desses compostos. O processo de sorção de herbicidas no solo influencia sua absorção pelas raízes das plantas e também os processos de dissipação, como degradação biológica, volatilização, lixiviação e transporte por erosão hídrica (Herwig et al., 2001).

A absorção dos herbicidas pelas plantas, a eficácia no controle das plantas daninhas e a movimentação no solo dependem, em grande parte, do equilíbrio entre os processos de sorção e dessorção. Os solos apresentam grande variabilidade nas características físicas, químicas e biológicas; é esperado que a natureza e a intensidade dos processos de sorção também sejam distintas. Nas condições tropicais, a maioria dos solos é bastante intemperizada e pobre em nutrientes, além de apresentar baixa capacidade de retenção, a qual depende do $\mathrm{pH}$ e do teor de matéria orgânica do solo (Fontes et al., 2001).

A sorção de herbicidas é muito influenciada pela matéria orgânica presente nos solos (Dick et al., 2010; Inoue et al., 2010). A matéria orgânica apresenta constituição bastante variada, sendo dividida em substâncias humificadas e não humificadas. A parte humificada é composta por ácidos húmicos, ácidos fúlvicos e humina, os quais representam a fração mais ativa na sorção de pesticidas (Farenhorst, 2006).

O diuron (N-(3,4-dichlorophenyl)-N, $\mathrm{N}$-dimethylurea) pertence ao grupo químico das ureias substituídas. É um herbicida de amplo espectro para o controle de mono e dicotiledôneas, aplicado em pré e pós-emergência das plantas daninhas, sendo registrado para as culturas de abacaxi, algodão, café, cana-de-açúcar e citros (Rodrigues \& Almeida, 2011). Por ser um herbicida não ionizável, permanece em sua forma molecular na solução do solo. Todavia, herbicidas não iônicos podem ser polares e, em virtude dessa condição, ser afetados pelo $\mathrm{pH}$ e adsorvidos aos complexos argilominerais e ao material orgânico do solo (Silva et al., 2007).

Considerando que existem poucos estudos sobre o comportamento de herbicidas em solos tropicais e que os resultados dessas pesquisas serão de grande importância para fazer recomendações seguras do ponto de vista técnico e ambiental, neste trabalho foi avaliado o comportamento sortivo e dessortivo do diuron em quatro latossolos.

\section{MATERIAL E MÉTODOS}

Os experimentos foram conduzidos no Laboratório de Herbicida no Solo da Universidade Federal de Viçosa. Foram utilizadas quatro amostras de latossolos de diferentes localidades: Latossolo Amarelo (LA) de Sooretama-ES; Latossolo VermelhoAmarelo (LVA) e Latossolo Vermelho-Amarelo húmico (LVAh) de Viçosa-MG; e Latossolo Vermelho (LV) de Três Marias-MG. As amostras dos solos foram coletadas na profundidade de 0 a $20 \mathrm{~cm}$. Para cada solo foram feitas curvas de neutralização de acidez com $\mathrm{CaCO}_{3}$, para posterior correção da acidez a valores de $\mathrm{pH}$ próximos a 6,0, com exceção do LA, cujo $\mathrm{pH}$ encontrava-se próximo de 6,0 em condições naturais. Posteriormente, trabalhou-se com os solos com o pH natural e, após a calagem, nas seguintes condições: $\mathrm{LVA}_{\mathrm{pH} 5,0} ;_{\mathrm{LVA}_{\mathrm{pH}}, 9}$; $\mathrm{LVAh}_{\mathrm{pH} 4,8} ; \mathrm{LVAh}_{\mathrm{pH} 5,8} ; \mathrm{LV}_{\mathrm{pH} 5,00} ; \mathrm{LV}_{\mathrm{pH} 6,2} ; \mathrm{e}$ $\mathrm{LA}_{\mathrm{pH} 6,30}$. As características químicas e físicas dos solos estudados estão apresentadas nas Tabelas 1 e 2, respectivamente.

A determinação do tempo necessário para o equilíbrio da sorção e a avaliação da sorção e dessorção do diuron nos solos foram realizadas de acordo com as recomendações da OECD (2000).

Para determinação do tempo de equilíbrio da sorção, solução contendo $10 \mathrm{mg} \mathrm{L}^{-1}$, obtida a partir de uma solução-estoque de $1.000 \mathrm{mg} \mathrm{L}^{-1}$ de diuron Pestanal ${ }^{\circledR}$ (Sigma Chem. Co, Padrão Analítico, 99,5\% de pureza), foi preparada em 
Tabela 1 - Resultados das análises químicas de amostras de latossolos antes e depois da correção do pH

\begin{tabular}{|c|c|c|c|c|c|c|c|c|c|c|c|}
\hline \multirow{2}{*}{ Solo } & \multirow{2}{*}{$\begin{array}{c}\mathrm{pH} \\
\left(\mathrm{H}_{2} \mathrm{O}\right) \\
\end{array}$} & $\mathrm{P}$ & $\mathrm{K}$ & $\mathrm{Ca}^{2+}$ & $\mathrm{Mg}^{2+}$ & $\mathrm{Al}^{3+}$ & $\mathrm{H}+\mathrm{Al}$ & $(\mathrm{t})$ & V & M & \multirow{2}{*}{$\frac{\mathrm{MO}}{\left(\operatorname{dag~kg}^{-1}\right)}$} \\
\hline & & \multicolumn{2}{|c|}{$\left(\mathrm{mg} \mathrm{dm}^{-3}\right)$} & \multicolumn{5}{|c|}{$\left(\mathrm{cmol}_{\mathrm{c}} \mathrm{dm}^{-3}\right)$} & \multicolumn{2}{|c|}{$(\%)$} & \\
\hline LVA & 5,0 & 3,5 & 50 & 0,8 & 0,3 & 0,8 & 8,91 & 2,18 & 13 & 37 & 3,7 \\
\hline LVA & 5,9 & 3,5 & 50 & 3,5 & 0,3 & 0,0 & 8,25 & 3,83 & 32 & 0 & 3,7 \\
\hline LVAh & 4,8 & 2,0 & 46 & 0,6 & 0,7 & 1,4 & 10,73 & 2,82 & 12 & 50 & 4,3 \\
\hline LVAh & 5,8 & 2,0 & 46 & 4,0 & 0,7 & 0,0 & 6,77 & 4,61 & 41 & 0 & 4,3 \\
\hline LV & 5,0 & 0,8 & 14 & 0,2 & 0,0 & 0,4 & 3,30 & 0,64 & 7 & 63 & 0,8 \\
\hline LV & 6,2 & 0,8 & 14 & 1,1 & 0,0 & 0,0 & 1,48 & 1,16 & 44 & 0 & 0,8 \\
\hline LA & 6,3 & 9,6 & 110 & 2,9 & 1,0 & 0,0 & 1,32 & 4,18 & 76 & 0 & 2,2 \\
\hline
\end{tabular}

Análises realizadas no Laboratório de Análises de Solo Viçosa, segundo a metodologia da Empresa Brasileira de Pesquisa Agropecuária Embrapa (1997); $(\mathrm{t})$ = capacidade de troca catiônica efetiva; $\mathrm{V}=$ saturação por bases; $\mathrm{m}=$ saturação por $\mathrm{Al}^{+3}$; $\mathrm{MO}=$ matéria orgânica.

Tabela 2 - Resultados das análises físicas e classificação textural de amostras de latossolos

\begin{tabular}{|l|c|c|c|c|c|}
\hline \multirow{2}{*}{ Solo } & A. grossa & A. fina & Silte & Argila & \multirow{2}{*}{ Classe textural } \\
\cline { 2 - 5 } & \multicolumn{5}{|c|}{$($ dag kg } \\
& -1 & \\
\hline LVA & 15 & 12 & 4 & 69 & Muito Argiloso \\
\hline LVAh & 23 & 13 & 5 & 59 & Muito Argiloso \\
\hline LV & 36 & 36 & 1 & 27 & Franco-Argiloarenoso \\
\hline LA & 60 & 19 & 1 & 20 & Franco-Arenoso \\
\hline
\end{tabular}

solução de $\mathrm{CaCl}_{2} \quad 0,01 \mathrm{~mol} \mathrm{~L} \mathrm{~L}^{-1}$. Em seguida, $10 \mathrm{~mL}$ da solução de $\mathrm{CaCl}_{2}$, contendo diuron, foram adicionados a tubos de polipropileno, os quais continham $2 \mathrm{~g}$ de solo. Os tubos com solução e solo foram devidamente vedados e colocados sob agitação vertical por diferentes tempos $(0 ; 1,0 ; 2,0 ; 4,0 ; 8,0 ; 12 ; 16 ; 20 ; 24$; e 30 horas) na temperatura de $27 \pm 2{ }^{\circ} \mathrm{C}$. Após agitação, as amostras foram centrifugadas a $2.260 \mathrm{~g}$, por sete minutos. Parte do sobrenadante foi filtrada em filtro Milipore com membrana PTFE de 0,45 $\mu \mathrm{m}$, para posterior análise por cromatografia liquida de alta eficiência (CLAE). Considerou-se como tempo de equilíbrio aquele a partir do qual a concentração da solução analisada permaneceu constante.

Para avaliar a sorção do diuron nos solos, foram preparadas soluções de trabalho a partir da solução-estoque nas concentrações de 0,5 , $1,2,4,8$ e $15 \mathrm{mg} \mathrm{L}^{-1}$ do herbicida em $\mathrm{CaCl}_{2}$ $0,01 \mathrm{~mol} \mathrm{~L}^{-1}$. Foram adicionados $10 \mathrm{~mL}$ dessas soluções em tubos de polipropileno contendo $2 \mathrm{~g}$ de solo. Em seguida, esses tubos foram colocados sob agitação em temperatura de $27 \pm 2{ }^{\circ} \mathrm{C}$ pelo tempo de equilíbrio, determinado anteriormente (12 horas). Após agitação, as amostras foram centrifugadas a $2.260 \mathrm{~g}$, por sete minutos. Parte do sobrenadante foi pipetada e filtrada em membrana PTFE de $0,45 \mu \mathrm{m}$, para posterior análise cromatográfica.

Os ensaios de dessorção foram realizados adicionando-se o mesmo volume de solução de $\mathrm{CaCl}_{2}$ 0,01 $\mathrm{mol} \mathrm{L}^{-1}$, isenta de herbicida, aos tubos que continham $8 \mathrm{mg} \mathrm{L}^{-1}$ de diuron, antes do ensaio de sorção. Esses tubos foram submetidos a nova agitação pelo mesmo tempo e temperatura em que foram realizados os ensaios de sorção. Após agitação, as amostras foram centrifugadas a $2.260 \mathrm{~g}$, por sete minutos. O sobrenadante foi totalmente retirado, sendo parte filtrada em membrana PTFE de $0,45 \mu \mathrm{m}$, para posterior análise cromatográfica. O procedimento de dessorção foi repetido por três vezes consecutivas $(12,24$ e 36 horas).

A determinação quantitativa do diuron foi feita em um sistema de cromatografia líquida de alta eficiência, modelo Shimadzu LC 20AT, com detector UV-Vis (Shimadzu SPD 20A) e coluna de aço inox (Shimadzu VP- ODS Shimpack $150 \mathrm{~mm} \times 4,6 \mathrm{~mm}$ d. i.). As condições cromatográficas para a análise foram fase móvel composta por água e acetonitrila na proporção de 50:50 (v/v), com fluxo de 1,2 $\mathrm{mL} \mathrm{min}^{-1}$, volume de injeção de $20 \mu \mathrm{L}$ e comprimento de onda de $254 \mathrm{~nm}$. O tempo de retenção do diuron nessas condições foi de aproximadamente sete minutos. A quantificação foi realizada por meio da comparação das áreas obtidas nos cromatogramas para cada ensaio, pelo método de calibração externa. A identificação, pelo tempo de retenção, foi feita mediante comparação com o padrão analítico do diuron. 
Calculou-se, em seguida, a quantidade de herbicida sorvido ao solo (Cs) em $\mathrm{mg} \mathrm{kg}^{1}$, pela diferença entre a quantidade de soluçãopadrão inicialmente adicionada ao solo $(\mathrm{Cp})$ em $\mathrm{mg} \mathrm{L} \mathrm{L}^{-1}$ e a quantidade encontrada na solução de equilíbrio (Ce) em $\mathrm{mg} \mathrm{L}^{-1}$. De posse dos valores de $\mathrm{Ce}$ e de $\mathrm{Cs}$, ajustou-se a equação de Freundlich $\left(\mathrm{Cs}=\mathrm{Kf} \mathrm{Ce}^{1 / \mathrm{n}}\right)$ para obtenção dos coeficientes de sorção, em que Kf e $1 / \mathrm{n}$ são constantes empíricas que representam a capacidade e intensidade de sorção, respectivamente.

O cálculo da quantidade do diuron dessorvido foi realizado pela diferença entre a concentração do herbicida no solo antes das etapas de dessorção e a concentração na solução analisada após cada intervalo avaliado. Posteriormente, calculou-se a porcentagem total de dessorção para cada intervalo de tempo (12, 24 e 36 horas).

As análises foram realizadas em triplicata, e os dados, submetidos à análise de regressão para interpretação dos resultados; os coeficientes das equações foram testados pelo teste t a 5\% de significância. Foram feitas análises de correlação de Pearson entre as propriedades dos solos estudados e os coeficientes de sorção.

\section{RESULTADOS E DISCUSSÃO}

O tempo de equilíbrio das estimativas das curvas de sorção do diuron aos solos está apresentado na Figura 1. Verifica-se que a cinética de sorção do diuron caracterizou-se por duas fases distintas: uma sorção rápida na fase inicial, nas quatro primeiras horas, seguida por uma fase lenta. Essa tendência foi observada em todos os solos avaliados. Esses resultados corroboram os de Liu et al. (2010), que atribuíram essa cinética de sorção do diuron ao fato de que no início há grande número de sítios de sorção disponíveis no solo e, na fase seguinte, os sítios superficiais vagos são dificeis de serem ocupados devido às forças de repulsão entre as moléculas do soluto na fase sólida e as moléculas em solução. Inoue et al. (2004) avaliaram a cinética de sorção do diuron em solos do Paraná e observaram que mais de $70 \%$ do diuron foi sorvido nas primeiras horas de contato do herbicida com os solos.
O tempo de equilíbrio foi atingido dentro de quatro horas no LVA e LVAh, independentemente do $\mathrm{pH}$ deles. No LA, o tempo foi de cinco horas, e no LV este foi influenciado pelo $\mathrm{pH}$. No pH 5,0 o tempo de equilíbrio foi de três horas, porém, quando o $\mathrm{pH}$ deste solo foi elevado a 6,2, observou-se o valor de sete horas. Nos estudos de sorção, foi selecionado o tempo 12 horas para todos os solos, pois a partir deste não se observou variação nas curvas de sorção do herbicida (Figura 1).

A isoterma de Freundlich ajustou-se adequadamente para descrever a sorção do diuron, como indicado pelos coeficientes de determinação das equações $\left(\mathrm{R}^{2}\right)$, que foram de 0,99 , nas curvas ajustadas de todos os solos (Tabela 3 e Figura 2). As isotermas ajustadas foram do tipo L, a qual apresenta inclinação não linear e côncava em relação à abscissa. Esse comportamento das curvas de sorção deve-se ao fato de o parâmetro $1 / n$ da equação, que determina a intensidade da sorção, ser menor que 1 (Giles et al., 1960). A taxa de sorção nos solos decresce com o aumento da concentração do diuron no solo, pois há diminuição da disponibilidade dos sítios de sorção deles. Os resultados encontrados estão de acordo com os de outros estudos, que demonstraram a adequação da equação de Freundlich

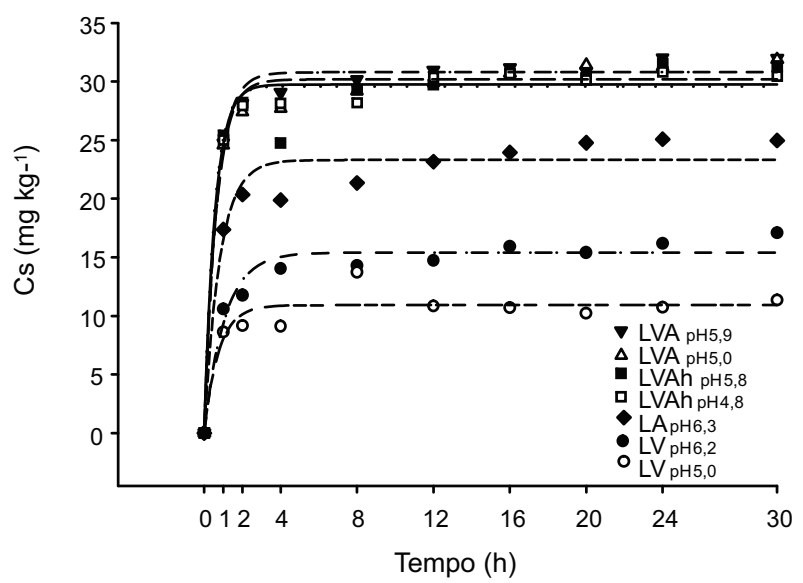

Figura 1 - Estimativas das curvas de cinética de sorção para o diuron em diferentes solos: $\mathrm{LVA}_{\mathrm{pH} 5,9} \hat{\mathrm{Y}}=30,7819(1-\exp$ $(-1,5356 x) R^{2}=0,99 ; \operatorname{LVA}_{\mathrm{pH} 5,0} \hat{\mathrm{Y}}=30,1771(1-\exp -1,5673 \mathrm{x})$ $\mathrm{R}^{2}=0,98 ; \mathrm{LVAh}_{\mathrm{pH} 4,8}=29,5826\left(1-\exp (1,8950 \mathrm{x}) \mathrm{R}^{2}=0,96 ;\right.$ $\mathrm{LVAh}_{\mathrm{pH} 5,8}=29,7298\left(1-\exp (-1,7679 \mathrm{x}) \mathrm{R}^{2}=0,98 ; \mathrm{LA}_{\mathrm{pH} 6,3}=\right.$ $23,2998\left(1-\exp (-1,2276 \mathrm{x}) \mathrm{R}^{2}=0,95 ; \mathrm{LV}_{\mathrm{pH} 5,0}=15,4024\right.$ $\left(1-\exp (-0,9325 \mathrm{x}) \mathrm{R}^{2}=0,96 ; \mathrm{e} \mathrm{LV}_{\mathrm{pH} 6.2}=10,9071\right.$ (1-exp $(-1,3240 \mathrm{x}) \mathrm{R}^{2}=0,89$, em função do tempo. 
Tabela 3 - Estimativas dos coeficientes de sorção (Kf e 1/n) e coeficientes de determinação $\left(\mathrm{R}^{2}\right)$ das isotermas de sorção do diuron em latossolos

\begin{tabular}{|c|c|c|c|c|c|c|c|}
\hline \multirow{2}{*}{ Coeficiente } & \multicolumn{7}{|c|}{ Solo } \\
\hline & $\mathrm{LVA}_{\mathrm{pH} 5,0}$ & $\mathrm{LVA}_{\mathrm{pH} 5,9}$ & LVAh $_{\mathrm{pH} 4,8}$ & LVA $_{\mathrm{pH} 5,8}$ & $\mathrm{LV}_{\mathrm{pH} 5,0}$ & $\mathrm{LV}_{\mathrm{pH} 6,2}$ & $\mathrm{LA}_{\mathrm{pH} 6,3}$ \\
\hline $\mathrm{Kf}$ & 14,37 & 15,27 & 13,43 & 14,13 & 1,79 & 3,85 & 7,14 \\
\hline $1 / \mathrm{n}$ & 0,78 & 0,76 & 0,81 & 0,83 & 0,72 & 0,83 & 0,78 \\
\hline $\mathrm{R}^{2}$ & 0,99 & 0,99 & 0,99 & 0,99 & 0,99 & 0,99 & 0,99 \\
\hline
\end{tabular}

LA - Latossolo Amarelo; LVAh - Latossolo Vermelho-Amarelo húmico; LVA - Latossolo Vermelho-Amarelo; LV - Latossolo Vermelho.
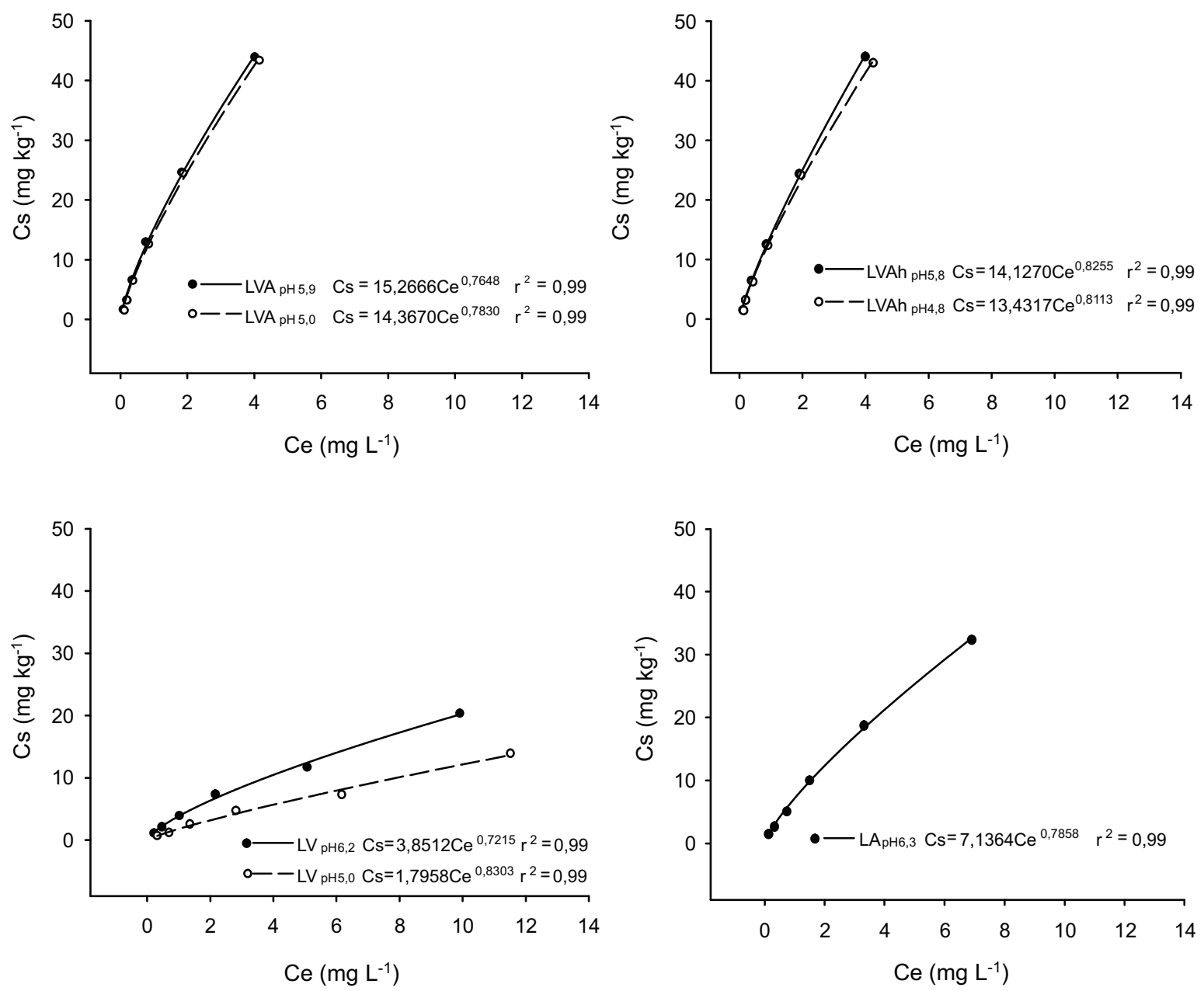

Figura 2 - Estimativas das isotermas de sorção nos solos: (A) Latossolo Vermelho-Amarelo (LVA); (B) Latossolo Vermelho-Amarelo húmico (LVAh); (C) Latossolo Vermelho (LV); (D) Latossolo Amarelo (LA).

para descrever a sorção do diuron nos solos (Inoue et al., 2006; Yu et al., 2006; Chaplain et al., 2008).

Os valores de Kf variaram de 1,79 a 15,26 (Figura 2 e Tabela 3). O LVA e LVAh apresentaram os maiores valores dos coeficientes de sorção do diuron, pois exibiram os maiores teores de matéria orgânica entre os solos estudados, favorecendo assim o processo de sorção. Esses resultados corroboram os de Boeira \& Souza (2004), os quais avaliaram a sorção do diuron em solos com textura contrastante e observaram que a sorção do herbicida foi bem menor no Neossolo Quartzarênico, quando comparado ao Latossolo Vermelho. 
Esses autores atribuíram essa maior sorção no latossolo ao fato de este apresentar teores mais elevados de carbono orgânico.

A elevação do $\mathrm{pH}$ em solos com maior capacidade de sorção (LVA e LVAh) praticamente não influenciou o $\mathrm{Kf}$ do diuron, pois esses solos apresentaram teores altos de argila e matéria orgânica e, consequentemente, há maior poder-tampão. Entretanto, no $\mathrm{LV}$, que apresentou os menores teores de matéria orgânica e argila, a elevação do $\mathrm{pH}$ contribuiu para o aumento da sorção do herbicida, pois em $\mathrm{pH}$ 5,0 o Kf foi de 1,79 e, com a elevação do $\mathrm{pH}$ para 6,2 , ele foi de 3,85 , praticamente dobrando esse parâmetro. O LA apresentou teor intermediário de matéria orgânica entre os solos avaliados; consequentemente, o Kf do herbicida neste solo foi de 7,13 , ficando entre o Latossolo Vermelho Amarelo e o Latossolo Vermelho (Tabela 3).

Correlações positivas foram observadas entre o Kf e a matéria orgânica $(r=0,96)$ e o teor de argila $(r=0,92)$, enfatizando assim a maior capacidade de sorção desse herbicida em solos com elevados teores de matéria orgânica e de argila. Correlação positiva também foi observada entre o $\mathrm{Kf}$ e a CTC efetiva $(r=0,66)$; não se observou correlação entre Kf e pH dos solos (Tabela 4).

O diuron é uma molécula não iônica, o que lhe confere características hidrofóbicas e justifica os maiores valores de Kf no solo com o teor mais elevado de matéria orgânica. A relação positiva entre teores de carbono orgânico e sorção desse herbicida é relatada por vários autores (Inoue et al., 2006; Alister et al., 2010; Liu et al., 2010). Na fração orgânica do solo há diferenças na capacidade de sorção entre seus constituintes. Ferri et al. (2005) isolaram a fração humina e os ácidos fúlvicos

Tabela 4 - Estimativas das correlações de Pearson entre os coeficientes de sorção do diuron e as características físicas e químicas dos solos

\begin{tabular}{|c|c|c|c|c|}
\hline \multirow{2}{*}{ Coeficiente } & \multicolumn{4}{|c|}{ Característica do solo } \\
\hline & $\operatorname{CTC}(\mathrm{t})^{\frac{1 /}{1}}$ & $\mathrm{MO}^{2 /}$ & $\mathrm{pH}$ & Argila \\
\hline $\mathrm{Kf}$ & $0,66^{*}$ & $0,96^{* *}$ & $-0,18^{\mathrm{ns}}$ & $0,92 * *$ \\
\hline $1 / \mathrm{n}$ & $0,34^{\mathrm{ns}}$ & $0,32^{\mathrm{ns}}$ & $0,34^{\mathrm{ns}}$ & $0,13^{\mathrm{ns}}$ \\
\hline
\end{tabular}

Planta Daninha, Viçosa-MG, v. 31, n. 1, p. 231-238, 2013 de um Argissolo Vermelho e observaram que a fração humina apresentou coeficiente de sorção dez vezes maior do que o dos ácidos húmicos para acetochlor, que, assim como o diuron, é um herbicida não iônico.

Gonzáles-Pradas et al. (1998) avaliaram o efeito da adição de turfa na sorção do diuron em solo calcário com pH 7,6. Em condições naturais, esse solo apresentava $0,18 \%$ de matéria orgânica e coeficiente de sorção de $2,17 \mathrm{mg} \mathrm{kg}^{-1}$. Quando o teor de matéria orgânica foi aumentado para $4,61 \%$ pela adição da turfa, a sorção do herbicida foi para $34,28 \mathrm{mg} \mathrm{kg}^{-1}$, demonstrando assim o efeito da matéria orgânica na sorção do diuron.

A porcentagem de dessorção do diuron foi inversa ao coeficiente de sorção (Kf), visto que solos que apresentaram os maiores Kf tiveram as menores taxas de dessorção. A sequência decrescente de dessorção observada foi $\mathrm{LV}_{\mathrm{pH} 5,0}>\mathrm{LV}_{\mathrm{pH} 6,2}>\mathrm{LA}_{\mathrm{pH} 6,3}>\mathrm{LVA}_{\mathrm{pH} 5,0}>\mathrm{LVA}_{\mathrm{pH} 5,9}$ $>\mathrm{LVAh}_{\mathrm{pH} 4,8}>\mathrm{LVAh}_{\mathrm{pH} 5,8}$ (Figura 3). A dessorção dos herbicidas é muito importante, pois determina a taxa de liberação e o potencial de mobilidade desses compostos nos solos. Os herbicidas com menor taxa de dessorção podem ter maior risco para as culturas em sucessão (Liu et al., 2010).

O LV apresentou elevada dessorção no tempo de 36 horas, com 50\% no pH 5,0 e 30\% no pH 6,2. Essas altas taxas nesse solo podem ser explicadas pelos baixos teores de matéria orgânica e argila, pois nessas condições a interação entre herbicida e solo pode ter sido constituída de interações fracas, como forças de van der Waals, o que favoreceu a maior dessorção do diuron. No LVA e LVAh, solos com elevados teores de matéria orgânica e argila, a dessorção do herbicida foi baixa: menor que $10 \%$. Essas taxas distintas de dessorção entre os solos significam que os mecanismos atuantes nos processos sortivos são diferentes em cada solo. É importante que seja considerado o fenômeno denominado histerese, que é a liberação pelo solo de apenas parte das moléculas anteriormente sorvidas, havendo assim resistência à dessorção distinta entre os solos. Em solos extremamente argilosos e com teores de matéria orgânica elevados, geralmente há alta sorção e baixa dessorção, o que diminui a disponibilidade do diuron na solução do solo. Contrariamente, solos com baixos teores de 

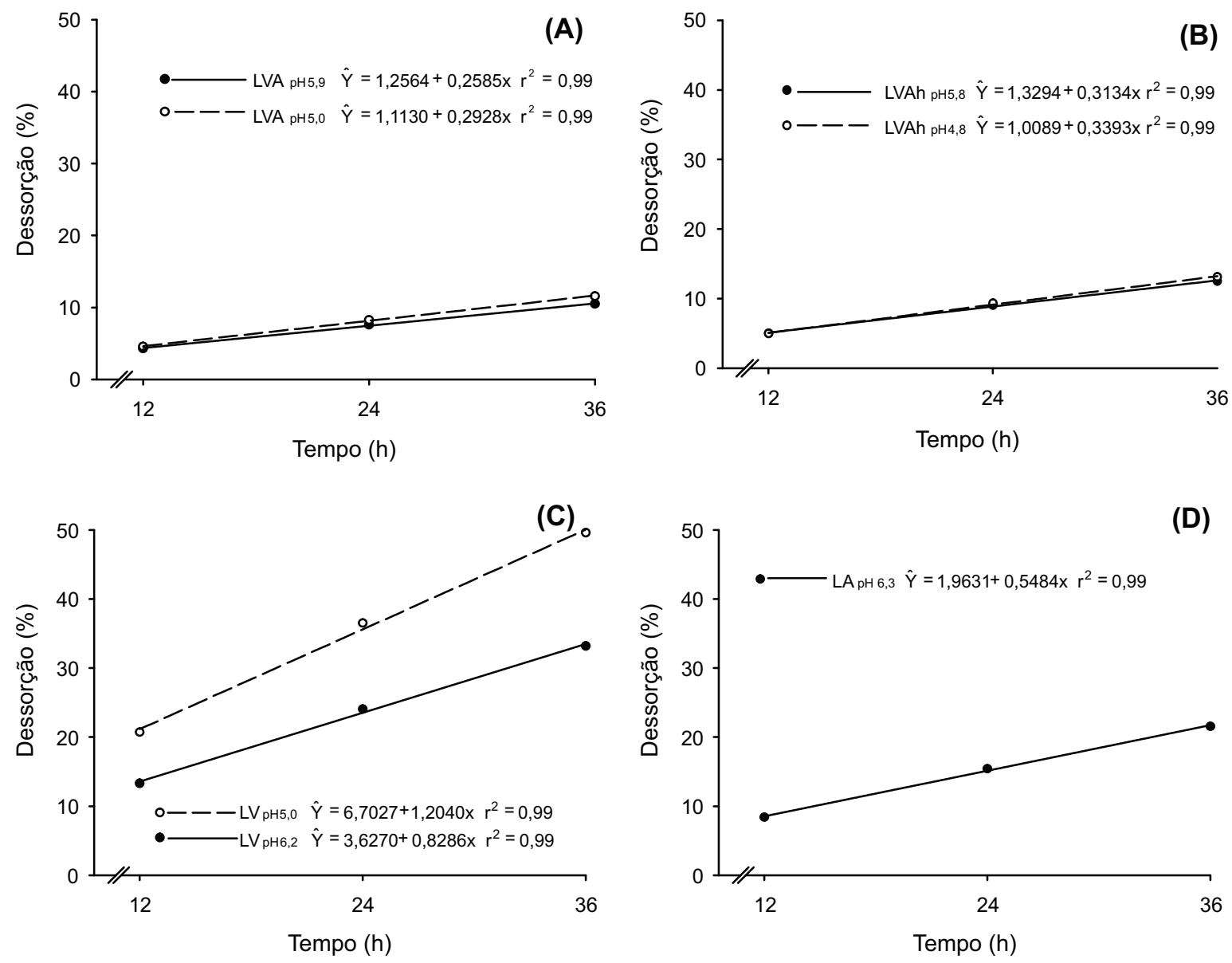

Figura 3 - Porcentagem de dessorção do diuron nos solos: (A) Latossolo Vermelho-Amarelo (LVA); (B) Latossolo Vermelho-Amarelo húmico (LVAh); (C) Latossolo Vermelho (LV); (D) Latossolo Amarelo (LA) ao longo do tempo.

matéria orgânica e argila, apresentam alta porcentagem de dessorção, o que pode favorecer uma maior movimentação do herbicida no perfil do solo.

O diuron apresenta persistência no solo, que varia de 30 a 365 dias (Giacomazzi \& Cochet, 2004). Em solos como o LV, que apresentou elevada dessorção, associada à relativamente longa persistência, o diuron pode ser carregado pelo fluxo de água do solo, podendo representar um risco de contaminação das águas subsuperficiais. Inoue et al. (2008) avaliaram a movimentação do diuron, por meio de bioensaio, em colunas de um Latossolo Vermelho de textura franco-arenosa, após aplicação de uma lâmina de água de $80 \mathrm{~mm}$, e observaram a movimentação do herbicida até $15 \mathrm{~cm}$ de profundidade. A movimentação do diuron no perfil do solo foi também observada por Dores et al. (2009) em condições de campo em Latossolo Amarelo de textura argiloarenosa, $\mathrm{pH} 5,1$ e carbono orgânico $1,5 \%$, com precipitação de $1.750 \mathrm{~mm}$ durante a condução do experimento. Esses autores detectaram a presença deste herbicida na profundidade de $50 \mathrm{~cm}$ em baixas concentrações, ou seja, $0,08 \%$ da dose aplicada. No entanto, observaram que as maiores perdas do diuron foram via escoamento superficial em torno de $14 \%$ do herbicida aplicado inicialmente, o que sugere risco de contaminação de águas superficiais por esse composto.

A sorção do diuron está diretamente relacionada aos teores de matéria orgânica, visto que solos com maiores teores de matéria orgânica, LVA e LVAh, apresentaram maiores coeficientes de sorção. O aumento do $\mathrm{pH}$ dos solos eleva a sorção do diuron de forma mais 
evidente no Latossolo Vermelho, sendo este o solo que apresentou os menores teores de matéria orgânica. O processo de dessorção do diuron foi inverso ao da sorção, pois solos com menor sorção apresentaram as maiores porcentagens de dessorção.

\section{AGRADECIMENTO}

Ao Conselho Nacional de Desenvolvimento Científico e Tecnológico $(\mathrm{CNPq})$, pelo apoio financeiro.

\section{LITERATURA CITADA}

ALISTER, C.; KOGAN, M. Rainfall effect on dissipation and movement of diuron and simazine in a vineyard soil. Planta Daninha, v. 28, p. 1059-1071, 2010. (Número Especial)

BACIGALUPO, M. A.; MERONI, G. Quantitative determination of diuron in ground and surface water by timeresolved fluoroimmunoassay: seasonal variations of diuron, carbofuran, and paraquat in an agricultural area. J. Agric. Food Chem., v. 55, n. 10, p. 3823-3828, 2007.

BOEIRA, R. C.; SOUZA, M. D. Sorção de diuron em solos com diferentes texturas. Jaguariúna: Embrapa Meio Ambiente, 2004. 5 p. (Circular Técnica, 9)

CHAPLAIN, V. et al. Soil hydrophobicity: a contribution of diuron sorption experiments. Europ. J. Soil Sci., v. 59, n. 6, p. $1202-1208,2008$

DICK, D. P. et al. Matéria orgânica em quatro tipos de solos brasileiros: composição química e sorção de atrazina. Química Nova, v. 33, n. 1, p. 14-19, 2010.

DORES, E. F. G. C. et al. Environmental behaviour of metolachlor and diuron in a tropical soil in the central region of Brazil. Water Air Soil Pollution, v. 197, n. 1-4, p. 175-183, 2009.

EMPRESA BRASILEIRA DE PESQUISA AGROPECUÁRIA - EMBRAPA. Centro Nacional de Pesquisa de Solos. Manual de métodos de análise de solos 2.ed. Rio de Janeiro: 1997. 212 p.

FARENHORST, A. Importance of soil organic matter fractions in soil-landscape and regional assessments of pesticide sorption and leaching in soil. Soil Sci. Soc. Am. J., v. 70, n. 3, p. 1005-1012, 2006.

FERRI, M. V. W. et al. Sorção do herbicida acetochlor em amostras de solo, ácidos húmicos e huminas de argissolo submetido à semeadura direta e ao preparo convencional. R. Bras. Ci. Solo, v. 29, n. 5, p. 705-714, 2005.

FONTES, M. P. F. et al. Eletroquímica das partículas coloidais e sua relação com a mineralogia de solos altamente intemperizados. Sci. Agric., v. 58, n. 3, p. 627-646, 2001.
GIACOMAZZI, S.; COCHET, N. Environmental impact of diuron transformation: a review. Chemosphere, v. 56, n. 11, p. 1021-1032, 2004

GILES, C. H. et al. A system of classification of solution adsorption isotherms. J. Chem. Soc., v. 111, n.1, p. $3973-3993,1960$

GONZÁLEZ-PRADAS, E. et al. Sorption and leaching of diuron on natural and peat-amended calcareous soil from spain. Water Res., v. 32, n. 9, p. 2814-2820, 1998.

HERWIG, U. et al. Physicochemical interactions between atrazine and clay minerals. Applied Clay Sci., v. 18, n. 5-6, p. 201-222, 2001.

INOUE, M. H. et al. Potencial de lixiviação de herbicidas utilizados na cultura do algodão em colunas de solo. Planta Daninha, v. 28, n. 4, p. 825-833, 2010

INOUE, M. H. et al. Lixiviação e degradação de diuron em dois solos de textura contrastante. Acta Sci. Agron., v. 30, n. 5 , p.631-638, 2008

INOUE, M. H. et al. Sorption kinetics of atrazine and diuron in soils from southern Brazil. J. Environ. Sci. Health. Part B. Pestic. Food Contam. Agric. Wastes, v. 39, n. 4, p. 589-601, 2004.

INOUE, M. H. et al. Sorption-desorption of atrazine and diuron in soils from southern Brazil. J. Environ. Sci. Health. Part B. Pestic. Food Contam. Agric. Wastes, v. 41, n. 5, p. 605-621, 2006.

LAPWORTH, D. J.; GOODDY, D. C. Source and persistence of pesticides in a semi-confined chalk aquifer of southeast England. Environ. Pollution, v. 144, n. 3, p. 1031-1044, 2006.

LIU, Y. et al. Adsorption and desorption behavior of herbicide diuron on various Chinese cultivated soils. J. Hazardous Mat., v. 178, n. 1-3, p. 462-468, 2010.

ORGANIZATION FOR ECONOMIC CO-OPERATION AND DEVELOPMENT - OECD. Guidelines for testing of chemicals: adsorption-desorption using a batch equilibrium method, 106. Paris: 2000. 44 p.

RODRIGUES, B. N.; ALMEIDA, F. S. Guia de herbicidas. 6.ed. Londrina: 2011. 697 p.

SILVA, A. A.; VIVIAN, R.; OLIVEIRA Jr., R. S. Herbicidas comportamento no solo. In: SILVA, A. A.; SILVA, J. F. Tópicos em manejo de plantas daninhas. Viçosa, $\mathrm{MG}$, Universidade Federal de Viçosa, 2007. 367 p.

SINDICATO NACIONAL DA INDÚSTRIA DE PRODUTOS PARA DEFESA AGRÍCOLA - SINDAG. Disponível em: <http://www.sindag.com.br/conexao/ anteriores/conexao_n22.pdf>. Acesso em: 3 maio 2011.

YU, X. Y.; YING, G. G; KOOKANA, R. S. Sorption and desorption behaviors of diuron in soils amended with charcoal. J. Agric. Food Chem., v. 54, n. 22, p. 8545-8550, 2006 